\section{Acute Cardiogenic Pulmonary Edema Mimicking Right Up- per Lobe Pneumonia}

\author{
Lars C Huber* and Line Dormond \\ Department of Internal Medicine, City Hospital Triemli, Zurich, Switzerland.
}

\begin{abstract}
We describe here the case of a patient with moderate heart failure and persistent mitral valve insufficiency following clipping that presented with acute onset of dyspnea, cough and respiratory failure. Chest X-ray at admission showed dense right-sided consolidations predominantely in the right upper lobe compatible with pneumonic infiltration. Polypragmatic treatment resulted in prompt radiographic resolution of these infiltrations demasking signs of pulmonary venous congestion and interstitial lung edema. This short case ancedote discusses the differential diagnosis and highlights the well-described but uncommon association between heart failure and unilateral pulmonary edema that might be confused with pneumonia.

Keywords: Heart failure, Mitral valve insufficiency, Pneumonia, Radiographic resolution, Unilateral pulmonary edema
\end{abstract}

\section{Case Anecdote}

An 88-year old man was admitted to our emergency department with acute onset of dyspnea, non-productive cough and respiratory failure. 6 weeks before presentation, the patient had undergone percutaneous mitral valve clipping (Panel A, arrow) for severe mitral valve insufficiency due to prolaps and flail posterior leaflet. The patient's history was remarkable for mitral valve endocarditis (viridians streptococci) more than ten years before clipping, coronary artery sclerosis without relveant stenosis, persistent atrial fibrillation and latent hypothyroidism. The patient's medication included, inter alia, oral anticoagulation with rivaroxaban for atrial fibrillation, loop diuretics (torasemid), a mineralocorticoid receptor antagonist (aldactone), an angiotensin-converting enzyme inhibitor (perindopril) a cardio-selective betablocking agent (bisoprolol) and proton pump inhibitor therapy (pantoprazol). Adherence to medication was reported to be good.

In the last 3 days before actual presentation, the patient suffered from repeated cough and felt feverish without having measured

*Corresponding author: Lars C Huber, Department of Internal Medicine, City Hospital Triemli, Zurich, Switzerland, Tel: +41 444161111; Email: lars.huber@triemli.zuerich.ch

Citation: Huber LC, Dormond L (2017) Acute Cardiogenic Pulmonary Edema Mimicking Right Upper Lobe Pneumonia. J Pulm Med Respir Res 3: 011.

Received: July 13, 2017; Accepted: August 23, 2017; Published: September 06, 2017 his temperature. In the night before admission, the patient awaked with acute orthopnea, non-productive cough and tachydyspnoea. At arrival in the emergency department, temperature was $36.7^{\circ} \mathrm{C}$, blood pressure $134 / 77 \mathrm{mmHg}$, pulse $119 / \mathrm{min}$ at irregular rate and oxygen saturation $96 \%(\mathrm{pO} 211.9 \mathrm{k} \mathrm{Pa})$ under $10 \mathrm{~L} / \mathrm{min}$ supplemental O2 delivered by an oxygen mask. Leukocytes and $\mathrm{C}$-reactive protein were normal (Lc 6.6 G/1, CRP 1.9 mg/l; normal Lc 3.6-10.5 G/1, CRP $<5$ $\mathrm{mg} / \mathrm{l}$ ), procalcitonin was not analysed. Levels of NT-pro-brain natriuretic peptide were markedly elevated $(3310 \mathrm{pg} / \mathrm{ml}$; normal $<300 \mathrm{pg} /$ $\mathrm{ml})$. Chest X-ray showed dense consolidations predominantely with in the right upper lobe compatible with pneumonic infiltration or unilateral pulmonary edema. Lateral film or computed tomography was not performed (Figure 1).

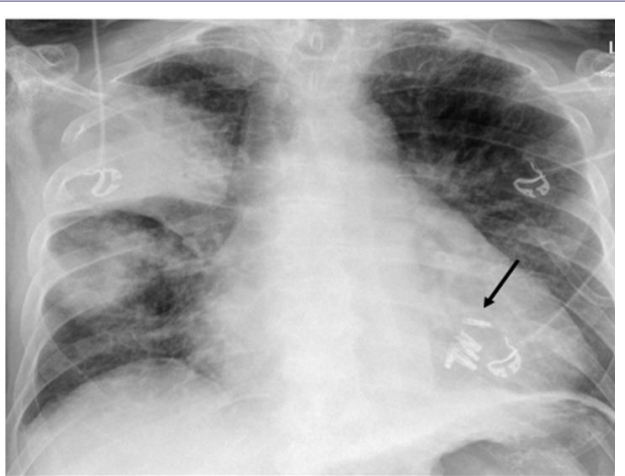

Figure 1: Chest radiograph findings. Chest $X$ ray at admission showing dense right-sided consolidations predominantely in the upper lobe. Mitral valve had been clipped (arrow) few weeks before the actual presentation.

The patient was transferred to our intensive care unit for cardiorespiratory monitoring and polypragmatic treatment including empirical antibiotic therapy (amoxicillin/clavulanic acid and clarithromycin for 5 days), a short trial of systemic steroids and transient ventilatory support with noninvasive ventilation, loop diuretics (furosemide) and inotropic agents (noradrenaline, milrion). Transthoracal echocardiography confirmed reduced systolic function of the left ventricle (LVEF 40-45\%) and persistent moderate-to-severe mitral valve disease with the presence of multiple regurgitation jets. Inflammatory markers, being normal at time of admission, peaked on day 2 (Lc $11.0 \mathrm{G} / 1$, CRP $121 \mathrm{mg} / \mathrm{l})$. Blood cultures remained sterile and urinary analysis for pneumococcal and Legionella antigen was negative.

The patient's condition improved continually under treatment. Follow-up X-ray on day 3 showed substantial resolution of the dense consolidation (Figure 2) demasking diffuse signs of pulmonary venous congestion and residual interstitial edema. Dose of loop diuretics was increased and the quinazoline derivative metolazone was added for a short period. As such, negative fluid balance could be achieved and the patient was dismissed to a rehabilitation facility on day 17 of hospitalization.

\section{Discussion}

Asymmetric pulmonary edema is an uncommon condition in patients with heart failure: it is observed in about $2 \%$ of patients presenting with acute cardiogenic edema and, in most cases, is associated 


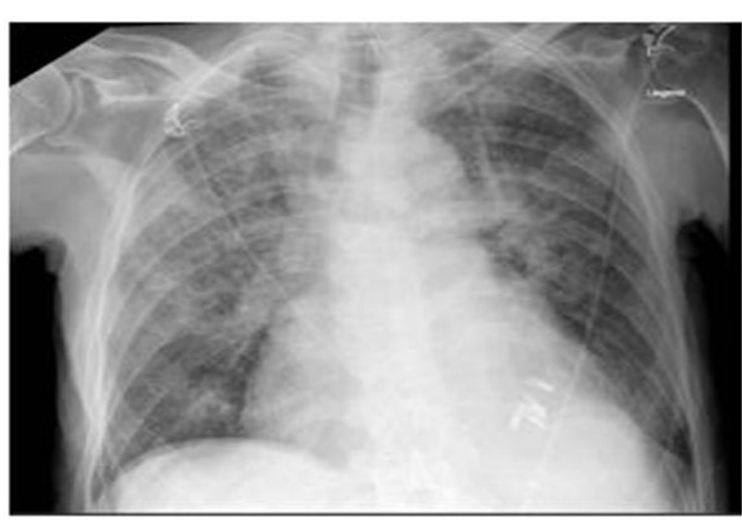

Figure 2: Chest radiograph findings. Follow-up chest radiograph 3 days after initial presentation showed diffuse signs of pulmonary venous congestion with residual interstitial edema but almost complete resolution of the dense consolidations.

with severe mitral valve insufficiency [1,2]. However, unilateral pulmonary edema has also been described in other conditions, for example in acute and chronic heart failure or in patients with leakage following mitral valve repair (reviewed in [2]). The predilection of unilateral pulmonary edema to the right side is explained by several mechanisms - in the context of mitral valve insufficiency it might be due to the anatomic relation of mitral valves and the pulmonary veins resulting in penetration of the thin-walled right upper pulmonary vein by the regurgitation jets [3].

In our case, inflammatory markers were within normal ranges at time of initial presentation but increased during further course. Normal levels of leukocytes and C-reactive protein decrease the likelihood for the diagnosis of pneumonia [4,5]. Conversely, increased levels of inflammatory markers, in particular C-reactive protein are well-described in patients with acute heart failure and, as such, have no discriminative value to distinguish between infection and venous congestion [6]. The diagnostic yield of procalcitonin, which was not assessed in our case, is of limited value when determined on top of $\mathrm{C}$-reactive protein to predict the diagnosis of pneumonia and adds no relevant clinical information in this setting as suggested by a recent diagnostic study [7].

Unilateral pulmonary edema might be mimicked by other conditions, i.e. pneumonia, aspiration, atelectasis, malignancy and pulmonary infarction following pulmonary embolism [8]. However, complete and rapid resolution under treatment is unlikely to occur in patients with pneumonic infiltrations, which, depending on comorbidities of the patient and type of microbiological pathogens, lag behind the clinical response and require longer time periods to resolve [9-12]. This is of particular relevance in elderly patients [12]. Cardiogenic pulmonary edema most commonly manifests in a bilateral perihilar pattern (for example in a "butterfly" or "bat wing" - like distribution) $[2,8]$. This short case vignette highlights how a failing heart syndrome might present as unilateral pulmonary edema and as such, can mimick the presentation of other conditions including pneumonia. Distinction between these entities is not always possible, in particular during early course of the disease and might result in polypragmatic treatment.

\section{Key Points}

Asymmetric pulmonary edema is observed in about $2 \%$ of patients with acute cardiogenic pulmonary edema and is most commonly associated with mitral valve regurgitation. Unilateral pulmonary edema might be confused with other conditions such as pneumonia, aspiration, atelectasis, malignancies and pulmonary infarction. Rapid radiographic clearance favors a diagnosis of unilateral edema while infiltrations due to pneumonia require more time to resolve completely.

\section{References}

1. Attias D, Mansencal N, Auvert B, Vieillard-Baron A, Delos A, et al. (2010) Prevalence, characteristics, and outcomes of patients presenting with cardiogenic unilateral pulmonary edema. Circulation 122: 1109-1115.

2. Myrianthefs P, Markou N, Gregorakos L (2011) Rare roentgenologic manifestations of pulmonary edema. Curr Opin Crit Care 17: 449-453.

3. Woolley K, Stark P (1999) Pulmonary parenchymal manifestations of mitral valve disease. Radiographics 19: 965-972.

4. Smith RP, Lipworth BJ (1995) C-reactive protein in simple community-acquired pneumonia. Chest 107: 1028-1031.

5. Ruiz-González A, Utrillo L, Bielsa S, Falguera M, Porcel JM (2016) The Diagnostic Value of Serum C-Reactive Protein for Identifying Pneumonia in Hospitalized Patients with Acute Respiratory Symptoms. J Biomark 2016: 2198745.

6. Pye M, Rae AP, Cobbe SM (1990) Study of serum C-reactive protein concentration in cardiac failure. Br Heart J 63: 228-230.

7. Van Vugt SF, Broekhuizen BDL, Lammens C, Zuithoff NPA, de Jong PA, et al. (2013) Use of serum $C$ reactive protein and procalcitonin concentrations in addition to symptoms and signs to predict pneumonia in patients presenting to primary care with acute cough: diagnostic study. BMJ 346: 2450 .

8. Bahl OP, Oliver GC, Rockoff SD, Parker BM (1971) Localized unilateral pulmonary edema: an unusual presentation of left heart failure. Chest 60 : 277-280.

9. Jay SJ, Johanson WG Jr, Pierce AK (1975) The radiographic resolution of Streptococcus pneumoniae pneumonia. N Engl J Med 293: 798-801.

10. Macfarlane JT, Miller AC, Roderick Smith WH, Morris AH, Rose DH (1984) Comparative radiographic features of community acquired Legionnaires' disease, pneumococcal pneumonia, mycoplasma pneumonia, and psittacosis. Thorax 39: 28-33.

11. Bruns AHW, Oosterheert JJ, Moussaoui El R, Opmeer BC, Hoepelman AIM, et al. (2010) Pneumonia recovery: discrepancies in perspectives of the radiologist, physician and patient. J Gen Intern Med 25: 203-206.

12. Solh El AA, Aquilina AT, Gunen H, Ramadan F (2004) Radiographic resolution of community-acquired bacterial pneumonia in the elderly. J Am Geriatr Soc 52: 224-229. 